\title{
A note on the elastic and geometric bounds for composite laminates
}

\author{
Paolo Vannucci \\ Université de Versailles et St Quentin, 45 Avenue des Etats-Unis, 78035 Versailles, France. \\ paolo.vannucci@uvsq.fr
}

February 5, 2012

\begin{abstract}
In this paper it is shown that a laminate composed of identical anisotropic layers, like for instance composite plies, cannot reach all the possible elastic states that are allowed by the classical elastic bounds on anisotropic constants. The analysis is carried on and facilitated by the use of particular tensor invariants, the so-called polar parameters.
\end{abstract}

\section{Introduction}

When some elastic layers are superposed and bonded together, they form a laminate. The stack so obtained is a structure whose elastic behaviour is ruled by three tensors, describing the in-plane, bending and coupled behaviour. When these tensors are normalized, they represent the elastic behaviour of a sort of fictitious material by which one could realize a single-layer plate, with the same overall thickness of the laminate and having the same in-plane, bending and coupled response of the laminate in all the directions.

It is well-known that the elastic constants of a given anisotropic material are bounded, because of the positive definiteness of the elastic energy. Such elastic bounds define an elastic domain of admissible values for the elastic constants.

We show here that when a laminate is fabricated bonding together identical layers of a given material, such an elastic domain is impossible to be entirely covered, because some more restrictive bounds are to be taken into account. These new bounds can be considered as geometrical bounds, because they are originated by some links that exist between the different elastic constants of the fictitious material and these links are due to the geometry of the stack, i.e. to the orientation of the layers and to their position in the stacking sequence. In other words, we show that the geometric domain is always smaller than and contained in the elastic domain. We propose also a degree of filling of the elastic domain by the geometric domain, which measures how much a laminate composed of a given material is far from covering all the elastic possible states. All the computations are developed in the same manner and separately for the tensors describing the in-plane and the bending properties. The case of the coupling tensor is not considered because it is not positive definite, actually it is not definite.

All the computations are done in a space of dimensionless invariant elastic constants, that can be introduced using the polar formalism. Its use greatly simplifies the analytical developments, basically for two reasons: on one side, it is based upon the use tensor invariants and gives a precise invariant statement of all the types of elastic symmetries and of the elastic 
bounds. On the other side, the polar formalism allows for reducing to the same space of invariant dimensionless parameters the elastic and geometrical bounds for the laminate's tensor. In addition, it offers a new physical interpretation of the classical lamination parameters too.

The paper is organized as follows: first, the basic equations, written using the polar formalism, are recalled; then, the elastic and geometric bounds are introduced for the case of orthotropic behaviour and rewritten in the space of suitable invariant dimensionless parameters. The proof that the geometric domain is always smaller than the elastic one is then given and discussed. Then, a measure of the degree of filling of the elastic domain is introduced as function of the type of the orthotropies of the basic layer and of the laminate's tensor and some numerical examples are given. Finally, the more involved case of non-orthotropic behaviour for the laminate's tensor is briefly treated and discussed. Some final remarks end the paper.

\section{Basic equations}

The in-plane, bending and coupling response of a laminate are given by the fundamental laminate's law, [4],

$$
\left\{\begin{array}{l}
\mathbf{N} \\
\mathbf{M}
\end{array}\right\}=\left[\begin{array}{ll}
\mathbf{A} & \mathbf{B} \\
\mathbf{B} & \mathbf{D}
\end{array}\right]\left\{\begin{array}{l}
\varepsilon \\
\kappa
\end{array}\right\},
$$

where $\mathbf{N}$ and $\mathbf{M}$ are respectively the in-plane forces and bending moments tensors, $\varepsilon$ is the in-plane strain tensor, $\kappa$ the curvature tensor, while the fourth-order elastic tensors $\mathbf{A}, \mathbf{B}$ and $\mathbf{D}$ describe respectively the extension, coupling and bending behaviour of the laminate.

The above three elastic tensors are usually normalized as follows:

$$
\mathbf{A}^{*}=\frac{\mathbf{A}}{h}, \quad \mathbf{B}^{*}=2 \frac{\mathbf{B}}{h^{2}}, \quad \mathbf{D}^{*}=12 \frac{\mathbf{D}}{h^{3}},
$$

$h$ being the total thickness of the laminate. In this way, the components of the three normalized tensors have the same dimensions, a force per unit of surface, so they can be compared. In addition, $\mathbf{A}^{*}$ and $\mathbf{D}^{*}$ can be considered as the elastic tensors of two fictitious materials by which one can imagine to construct a single layer plate with thickness $h$, having the same elastic response of the laminate, respectively in extension and bending, for all the directions. As a consequence, the components of $\mathbf{A}^{*}$ and $\mathbf{D}^{*}$, that are, generally speaking, anisotropic, must satisfy some conditions in order to satisfy the requirement of positive definiteness, just like any other elastic tensor. Such conditions bound an elastic domain containing the admissible values of the elastic parameters.

The case of $\mathbf{B}^{*}$ is different, because it is not positive definite, actually it is simply not definite. In addition, the idea of a fictitious material for the coupled response has not a precise and direct mechanical meaning; for these reasons, the case of the coupling tensor will not be considered in the following.

Tensors $\mathbf{A}^{*}$ and $\mathbf{D}^{*}$ are given by composition laws, that in the case, assumed in the rest of the paper, of identical layers, are

$$
\begin{aligned}
& \mathbf{A}^{*}=\frac{1}{n} \sum_{j=1}^{n} \mathbf{Q}\left(\delta_{j}\right), \\
& \mathbf{D}^{*}=\frac{1}{n^{3}} \sum_{j=1}^{n} d_{j} \mathbf{Q}\left(\delta_{j}\right) .
\end{aligned}
$$


In eq. (3), $n$ is the number of the layers, $\delta_{j}$ is the orientation of the $j$-th layer, $\mathbf{Q}$ is the reduced stiffness tensor of the basic layer and the coefficients $d_{j}$ are given by

$$
d_{j}=12 j(j-n-1)+4+3 n(n+2) \text {. }
$$

To be remarked that the sum of the coefficients $d_{j}$ over the layers is equal to $n^{3}$. The above composition laws, eq. (3), are independent from the tensor representation; so they can be applied not only to the Cartesian components, but also to any other tensor components, in particular to tensor invariants.

The use of tensor invariants can be very profitable in some situations; in particular, with the elastic tensor of an anisotropic material, the use of invariants is interesting, because they are frame independent quantities, hence they represent intrinsic elastic properties. Among the different possible choices of tensor representation, we adopt in the rest of the paper the polar formalism, introduced as early as 1979 by G. Verchery for bi-dimensional tensors, 13. The polar invariants are directly linked to the elastic symmetries, allow for a simple expression of the elastic properties in a rotated frame and of the elastic bounds by invariants. A deeper insight in the matter can be found in [9], here only the essential points for the rest of the paper are briefly recalled.

The six Cartesian components of the tensor $\mathbf{Q}\left(\delta_{j}\right)$ can be given as function of six other parameters, the polar components, in the following way:

$$
\left\{\begin{array}{l}
Q_{x x x x}\left(\delta_{j}\right)=T_{0}^{L}+2 T_{1}^{L}+R_{0}^{L} \cos 4\left(\Phi_{0}^{L}-\delta_{j}\right)+4 R_{1}^{L} \cos 2\left(\Phi_{1}^{L}-\delta_{j}\right), \\
Q_{x x x y}\left(\delta_{j}\right)=R_{0}^{L} \sin 4\left(\Phi_{0}^{L}-\delta_{j}\right)+2 R_{1}^{L} \sin 2\left(\Phi_{1}^{L}-\delta_{j}\right), \\
Q_{x x y y}\left(\delta_{j}\right)=-T_{0}^{L}+2 T_{1}^{L}-R_{0}^{L} \cos 4\left(\Phi_{0}^{L}-\delta_{j}\right), \\
Q_{x y x y}\left(\delta_{j}\right)=T_{0}^{L}-R_{0}^{L} \cos 4\left(\Phi_{0}^{L}-\delta_{j}\right), \\
Q_{x y y y}\left(\delta_{j}\right)=-R_{0}^{L} \sin 4\left(\Phi_{0}^{L}-\delta_{j}\right)+2 R_{1}^{L} \sin 2\left(\Phi_{1}^{L}-\delta_{j}\right), \\
Q_{y y y y}\left(\delta_{j}\right)=T_{0}^{L}+2 T_{1}^{L}+R_{0}^{L} \cos 4\left(\Phi_{0}^{L}-\delta_{j}\right)-4 R_{1}^{L} \cos 2\left(\Phi_{1}^{L}-\delta_{j}\right) .
\end{array}\right.
$$

The four polar components $T_{0}^{L}, T_{1}^{L}, R_{0}^{L}$ and $R_{1}^{L}$ are invariant elastic moduli, while the two polar parameters $\Phi_{0}^{L}$ and $\Phi_{1}^{L}$ are angles. The difference of the two polar angles, $\Phi_{0}^{L}-\Phi_{1}^{L}$, is the fifth independent tensor invariant of $\mathbf{Q}$. The superscript $L$ stands for layer and denotes all the polar quantities relative to the basic layer.

The inversion of eqs. (5) gives the expression of the polar parameters as functions of the Cartesian components, here written, for the sake of clarity, in the $x-y$ frame, i.e. for $\delta_{j}=0$ :

$$
\left\{\begin{array}{l}
8 T_{0}^{L}=Q_{x x x x}-2 Q_{x x y y}+4 Q_{x y x y}+Q_{y y y y} \\
8 T_{1}^{L}=Q_{x x x x}+2 Q_{x x y y}+Q_{y y y y} \\
8 R_{0}^{L} e^{4 i \Phi_{0}^{L}}=Q_{x x x x}-2 Q_{x x y y}-4 Q_{x y x y}+Q_{y y y y}+4 i\left(Q_{x x x y}-Q_{x y y y}\right) \\
8 R_{1}^{L} e^{2 i \Phi_{1}^{L}}=Q_{x x x x}-Q_{y y y y}+2 i\left(Q_{x x x y}+Q_{x y y y y}\right)
\end{array}\right.
$$

The polar invariants are linked to the elastic symmetries; it has been shown that ordinary orthotropy is defined by the condition

$$
\Phi_{0}^{L}-\Phi_{1}^{L}=K^{L} \frac{\pi}{4}, \quad K^{L} \in\{0,1\}
$$

while two special orthotropies exist and are defined by the two independent conditions

$$
\begin{aligned}
& R_{0}^{L}=0, \\
& R_{1}^{L}=0 .
\end{aligned}
$$


The fact that two values are possible for $K^{L}$ in eq. (7) means that actually two different types of ordinary orthotropy can exist for the same set of the invariant moduli $T_{0}, T_{1}, R_{0}$ and $R_{1}$. The influence of this parameter on the elastic behaviour, namely on the solution to some optimal problems, has been discussed in [10]. The special orthotropy determined by eq. (8) is named $R_{0}$-orthotropy and is deeply analysed in [8] and [11. The special orthotropy determined by eq. (9) is the classical case of the so-called square symmetry, i.e. the bidimensional corresponding of the cubic syngony: all the quantities are periodic of $\pi / 2$.

As it is immediately recognized looking at eq. (5), the two polar invariants $T_{0}^{L}$ and $T_{1}^{L}$ determine the entire isotropic part of $\mathbf{Q}$, while $R_{0}^{L}, R_{1}^{L}, \Phi_{0}^{L}$ and $\Phi_{1}^{L}$ affect the anisotropic part only. Then, isotropy is simply obtained when eqs. (8) and (9) are satisfied at the same time: isotropy corresponds to the simultaneous presence of the two special orthotropies.

A frame can be fixed by choosing a value for one of the two polar angles; normally the choice $\Phi_{1}^{L}=0$ is done; for usual unidirectional plies, this choice corresponds to putting the $x$-axis of the material frame in the direction of the fibres.

The polar representation stated in eq. (5) for tensor Q, applies as well to any other bidimensional elastic tensor, hence to $\mathbf{A}^{*}$ and $\mathbf{D}^{*}$ too. Let us denote in the following by $T_{0}$, $T_{1}, R_{0}$ and $R_{1}, \Phi_{0}$ and $\Phi_{1}$ the polar parameters of $\mathbf{A}^{*}$ or $\mathbf{D}^{*}$. Applying the composition laws (3) to the polar components of $\mathbf{A}^{*}$ or $\mathbf{D}^{*}$ gives then, [12],

$$
\left\{\begin{array}{l}
T_{0}=T_{0}^{L}, \\
T_{1}=T_{1}^{L}, \\
R_{0} e^{4 i \Phi_{0}}=R_{0}^{L} e^{4 i \Phi_{0}^{L}}\left(\xi_{0}+i \xi_{2}\right), \\
R_{1} e^{2 i \Phi_{1}}=R_{1}^{L} e^{2 i \Phi_{1}^{L}}\left(\xi_{1}+i \xi_{3}\right) .
\end{array}\right.
$$

In eq. (10) $\xi_{1}$ to $\xi_{4}$ are the so-called lamination parameters, introduced first by Tsai and Hahn in 1980, [7], defined as

$$
\left\{\begin{array}{l}
\xi_{0}+i \xi_{2}=\frac{1}{n} \sum_{j=1}^{n} e^{4 i \delta_{j}} \\
\xi_{1}+i \xi_{3}=\frac{1}{n} \sum_{j=1}^{n} e^{2 i \delta_{j}}
\end{array}\right.
$$

for tensor $\mathbf{A}^{*}$, and as

$$
\left\{\begin{array}{l}
\xi_{0}+i \xi_{2}=\frac{1}{n^{3}} \sum_{j=1}^{n} d_{j} e^{4 i \delta_{j}}, \\
\xi_{1}+i \xi_{3}=\frac{1}{n^{3}} \sum_{j=1}^{n} d_{j} e^{2 i \delta_{j}},
\end{array}\right.
$$

for tensor $\mathbf{D}^{*}$. Though defined in a slightly different way, the lamination parameters of $\mathbf{A}^{*}$ and $\mathbf{D}^{*}$ must satisfy some limitations that are the same in both the cases; these limitations are specified in the next section.

It is apparent, looking at eq. 10 , that the isotropic part of $\mathbf{A}^{*}$ or $\mathbf{D}^{*}$ is just that of $\mathbf{Q}$, i.e. of the basic layer, while the anisotropic part changes, though $R_{0}$ is affected only by $R_{0}^{L}$ and $R_{1}$ only by $R_{1}^{L}$. This has a certain importance for the following developments.

Without loosing in generality, some simplifications can be made in eq. (10). In fact, all the layers are actually orthotropic, so eq. (7) can be used. In addition, as previously specified, the frame is fixed choosing a value for one of the two polar angles. So, once and for all, we put

$$
\Phi_{1}^{L}=\Phi_{1}=0
$$

Finally, we consider for the while the case of ordinarily orthotropic $\mathbf{A}^{*}$ or $\mathbf{D}^{*}$, when

$$
\xi_{2}=\xi_{3}=0,
$$


because, by eqs. (13) and (7), the imaginary parts of eqs. $(10,3)$ are null; the more general case of complete anisotropy will be considered later on. In this way, eqs. $(10,4)$ become

$$
\left\{\begin{array}{l}
(-1)^{K} R_{0}=(-1)^{K^{L}} R_{0}^{L} \xi_{0}, \\
R_{1}=R_{1}^{L} \xi_{1} .
\end{array}\right.
$$

\section{$3 \quad$ Elastic and geometrical bounds}

Let us now consider the bounds that must be satisfied by the quantities introduced above. These are of two types: the elastic and geometrical bounds.

Elastic bounds are the result of the positive definiteness of the strain energy: tensors $\mathbf{A}^{*}$ and $\mathbf{D}^{*}$ must be positive definite. For anisotropic tensors, normally these bounds concern the Cartesian components and are rather complicate. But with the polar formalism, the bounds are rather simple and concern invariant quantities. For an orthotropic tensor, the bounds are, [9],

$$
\left\{\begin{array}{l}
T_{0}-R_{0}>0 \\
T_{1}\left[T_{0}+(-1)^{K} R_{0}\right]-2 R_{1}^{2}>0 .
\end{array}\right.
$$

In addition, the parameters $R_{0}$ and $R_{1}$ can be interpreted as norms of complex quantities, eqs. (6) so they are non negative:

$$
\left\{\begin{array}{l}
R_{0} \geq 0 \\
R_{1} \geq 0
\end{array}\right.
$$

Of course, eqs. (16) and (17) can be written not only for $\mathbf{A}^{*}$ and $\mathbf{D}^{*}$, but also for $\mathbf{Q}$, simply adding the superscript $L$ to all the quantities.

Let us now consider the geometric bounds, i.e. those that are inherent to the construction of the stack. First of all, taking the norms of eqs. $(10,4)$ one gets

$$
\left\{\begin{array}{l}
R_{0}=R_{0}^{L} \sqrt{\xi_{0}^{2}+\xi_{2}^{2}} \\
R_{1}=R_{1}^{L} \sqrt{\xi_{1}^{2}+\xi_{3}^{2}} .
\end{array}\right.
$$

It can be proved, see [2], that the following bounds exist, in the most general case, for the lamination parameters of $\mathbf{A}^{*}$ or $\mathbf{D}^{*}$ :

$$
\left\{\begin{array}{l}
2 \xi_{1}^{2}\left(1-\xi_{0}\right)+2 \xi_{3}^{2}\left(1+\xi_{0}\right)+\xi_{0}^{2}+\xi_{2}^{2}-4 \xi_{1} \xi_{2} \xi_{3} \leq 1 \\
\xi_{1}^{2}+\xi_{3}^{2} \leq 1 \\
-1 \leq \xi_{0} \leq 1
\end{array}\right.
$$

These bounds, concerning purely geometric quantities, i.e. combinations of the circular functions of the layer orientations, come from the trigonometric nature of the quantities involved in the definition of the lamination parameters, eqs. (11) and (12), along with the fact, for $\mathrm{D}^{*}$, that the sum of the coefficients $d_{j}$ is $n^{3}$.

It is sufficient a quick glance at eqs. (11) and (12) to check that eq. 192 ) is valid also for $\xi_{0}$ and $\xi_{2}$ :

$$
\xi_{0}^{2}+\xi_{2}^{2} \leq 1
$$

So, through eqs. 18), 192) and (20) we get the bounds:

$$
\left\{\begin{array}{l}
R_{0} \leq R_{0}^{L} \\
R_{1} \leq R_{1}^{L}
\end{array}\right.
$$


Finally, in the case of $\mathbf{A}^{*}$ or $\mathbf{D}^{*}$ orthotropic, the bounds in eq. (19) become, through eq. (14),

$$
\left\{\begin{array}{l}
2 \xi_{1}^{2}\left(1-\xi_{0}\right)+\xi_{0}^{2} \leq 1 \\
\xi_{1}^{2} \leq 1 \\
-1 \leq \xi_{0} \leq 1
\end{array}\right.
$$

that are usually written in the equivalent form, [5], [6],

$$
\left\{\begin{array}{l}
-1 \leq \xi_{1} \leq 1 \\
2 \xi_{1}^{2}-1 \leq \xi_{0} \leq 1
\end{array}\right.
$$

Actually, from eqs. $(152)$ and $\left(17_{2}\right)$ it follows that $\xi_{1}$ in $(23)$ must be limited to non negative values. As it is suddenly recognized from eqs. (11) or (12), negative values of $\xi_{1}$ correspond to stacking sequences where the angle $\pi / 2$ have been added to all the orientations, i.e. to a rotation of the laminate of $-\pi / 2$, which is meaningless. Finally, the bounds to be taken into account are

$$
\left\{\begin{array}{l}
R_{0}<T_{0}, \\
2 R_{1}^{2}<T_{1}\left[T_{0}+(-1)^{K} R_{0}\right], \\
0 \leq R_{0} \\
0 \leq R_{1} \\
R_{0} \leq R_{0}^{L} \\
R_{1} \leq R_{1}^{L} \\
0 \leq \xi_{1} \\
\xi_{1} \leq 1 \\
2 \xi_{1}^{2}-1 \leq \xi_{0} \\
\xi_{0} \leq 1
\end{array}\right.
$$

The first four bounds in (24) are elastic bounds, while the other are geometric bounds. These bounds, that are written for a part in the space of the elastic invariants, and for the other part in the space of the lamination parameters, can all be written in a space of adimensional invariant parameters of $\mathbf{A}^{*}$, or $\mathbf{D}^{*}$, and $\mathbf{Q}$. This will first reduce the number of independent bounds in eq. (24), secondly will allow to put in direct relation the invariants of $\mathbf{A}^{*}$, or $\mathbf{D}^{*}$, with those of $\mathbf{Q}$ and finally will let appear explicitly the type of orthotropy of the basic layer and that of the laminate.

\section{Introducing dimensionless invariant parameters}

Let us introduce the following dimensionless quantities:

$$
\rho=\frac{R_{0}^{L}}{R_{1}^{L}}, \quad \rho_{0}=\frac{R_{0}}{R_{0}^{L}}, \quad \rho_{1}=\frac{R_{1}}{R_{1}^{L}}, \quad \tau_{0}=\frac{T_{0}^{L}}{R_{0}^{L}}, \quad \tau_{1}=\frac{T_{1}^{L}}{R_{1}^{L}} .
$$

Among them, $\rho, \tau_{0}$ and $\tau_{1}$ concern the basic layer, hence the material composing the laminate, while $\rho_{0}$ and $\rho_{1}$ have at the numerator a quantity concerning the laminate, while at the denominator one regarding the material. All of them are invariant quantities, as ratios of invariant parameters. In addition, thanks to the fact that $R_{0}$ and $R_{1}$ are non negative quantities and through eq. 24,2$)$, it is also

$$
\rho>0, \quad \rho_{0}>0, \quad \rho_{1}>0, \quad \tau_{0}>1, \quad \tau_{1}>0 .
$$


Using eq. (25) into eq. (15), we obtain the following expressions for the lamination parameters $\xi_{0}$ and $\xi_{1}$ :

$$
\left\{\begin{array}{l}
\xi_{0}=(-1)^{K-K^{L}} \rho_{0} \\
\xi_{1}=\rho_{1}
\end{array}\right.
$$

This result gives, on one side, a new interpretation of the lamination parameters, at least for the orthotropic case: they are the ratios of homologous polar invariants for the laminate and for the material. On the other side, it makes appear explicitly the orthotropy types of the laminate and of the material, specified by $K$ and $K^{L}$ respectively.

After inserting eqs. (25) and (27) into eq. (24) and remembering that the isotropic part of $\mathbf{A}^{*}$ or $\mathbf{D}^{*}$ is just identical to that of the material, eq. $\left({ }_{10}, 2\right)$, we obtain the dimensionless and invariant version of bounds $(24)$ :

$$
\left\{\begin{array}{l}
\rho_{0}<\tau_{0}, \\
2 \rho_{1}^{2}<\rho \tau_{1}\left[\tau_{0}+(-1)^{K} \rho_{0}\right] \\
0 \leq \rho_{0} \\
0 \leq \rho_{1} \\
\rho_{0} \leq 1 \\
\rho_{1} \leq 1 \\
0 \leq \rho_{1} \\
\rho_{1} \leq 1 \\
2 \rho_{1}^{2}-1 \leq(-1)^{K-K^{L}} \rho_{0}, \\
(-1)^{K-K^{L}} \rho_{0} \leq 1
\end{array}\right.
$$

It is apparent that some of the bounds $(28)$ are redundant: eqs. $(28,7)$ are the same, while it is immediately recognized that eq. $\left(28_{10}\right)$, thanks to $\left(28_{3}\right)$, is never more restrictive than $(28)$, so it can be discarded. Also the identical eqs. $(28,8)$ can be eliminated, because such condition is never more restrictive than that given by eqs. $\left(28_{, 9}\right)$. Finally, also $\left(28_{1}\right)$ can be eliminated, because, by eq. $\left(26_{4}\right)$, it is always less restrictive than $\left(28_{5}\right)$.

It is interesting to notice that this last result has been obtained because, though eq. $\left(21_{1}\right)$ concerns the polar invariants of the laminate, the isotropic invariant $T_{0}$ is exactly equal to the corresponding one of the material, $T_{0}^{L}$, see eq. $\left(10_{1}\right)$. So, it can replace $T_{0}$ in $\left(10_{1}\right)$, which divided by $R_{0}^{L}$ gives eq. (281). As $\tau_{0}$ is an invariant parameter concerning a real material, condition $\left(26_{4}\right)$ is necessarily satisfied, which allows for discarding the bound $\left(28_{1}\right)$. This is one of the advantages of using the polar formalism.

Finally, the dimensionless independent bounds are

$$
\left\{\begin{array}{l}
0 \leq \rho_{0} \\
0 \leq \rho_{1} \\
\rho_{0} \leq 1 \\
2 \rho_{1}^{2}-1 \leq(-1)^{K-K^{L}} \rho_{0} \\
\frac{2 \rho_{1}^{2}}{\rho \tau_{1}}-\tau_{0}<(-1)^{K} \rho_{0} .
\end{array}\right.
$$

\section{The geometric and elastic domains}

In the sector $\rho_{1} \geq 0, \rho_{0} \geq 0$, eq. $(29)$ and $(29$ ), two parabolas, define each one a sub-domain, together with the other conditions in eq. (29). We show in this section that the sub-domain defined by eq. $(29)$, a geometric condition, is always smaller than and contained in that 
defined by eq. $(29$ ), an elastic condition. Conventionally, to have a finite elastic domain, whenever $K=0$ the condition $(29$, ) is also used to bound the elastic domain, otherwise unbounded.

The parabolas defined by eqs. $\left(29_{5,6}\right)$ depend on the parameters $K^{L}$ and $K$, i.e. on the type of ordinary orthotropies of the material and of the laminate. We want hence to show that the only possible four situations are those described in Fig. 1, where G stands for geometric and denotes the parabola $(294)$, while E stands for elastic and denotes the parabola $(29)$.
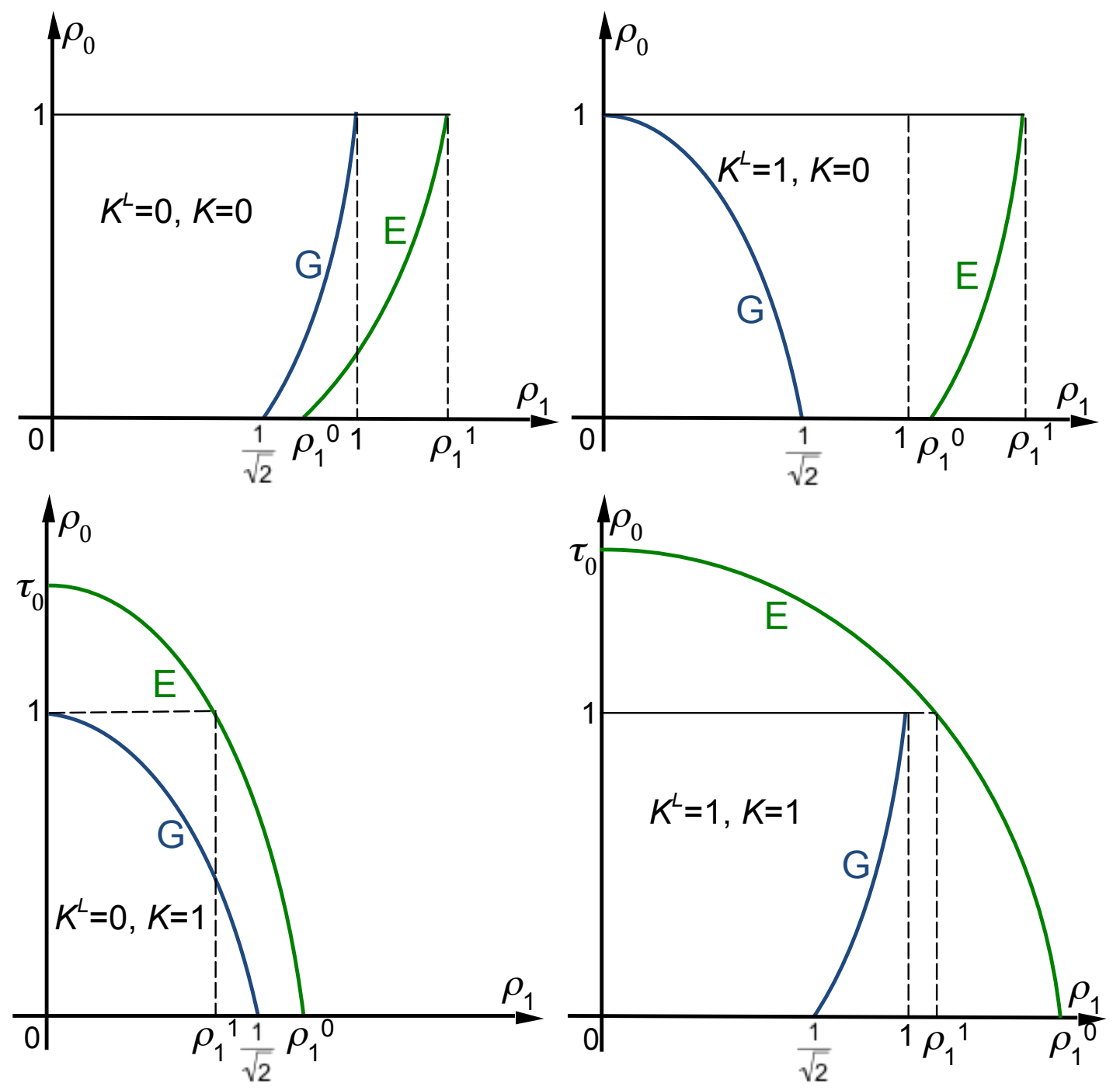

Fig. 1 The four possible cases of geometric and elastic domains.

Let us denote by $\rho_{1}^{0}$ the intersection of parabola $\mathrm{E}$ with the axis $\rho_{0}=0$ and by $\rho_{1}^{1}$ its intersection with the straight line $\rho_{0}=1$; through eq. $(29$ ) we have

$$
\rho_{1}^{0}=\sqrt{\frac{\rho \tau_{0} \tau_{1}}{2}}, \quad \rho_{1}^{1}=\sqrt{\frac{1}{2} \rho \tau_{1}\left(\tau_{0}+(-1)^{K}\right)} .
$$

Then, to show that the only possible situations are those represented in Fig. 1, it is sufficient to show that

$$
\left\{\begin{array}{l}
\tau_{0}>1, \\
\rho_{1}^{0}>\frac{1}{\sqrt{2}}, \\
\rho_{1}^{1}>1
\end{array}\right.
$$


Putting eq. (30) into conditions (31) gives

$$
\left\{\begin{array}{l}
\tau_{0}>1 \\
\rho \tau_{0} \tau_{1}>1 \\
\rho \tau_{1}\left(\tau_{0}+1\right)>2 \text { for } K=0 \\
\rho \tau_{1}\left(\tau_{0}-1\right)>2 \text { for } K=1
\end{array}\right.
$$

The conditions in eq. (32) concern only invariant dimensionless parameters of the material, hence they do not depend on the stacking sequence. Moreover, all of them are always satisfied. In fact, using the definition of the dimensionless parameters, eq. (25), it is easily checked that conditions $(32,4)$ are actually the bounds in eq. (16), always satisfied for any material with $K=1$. After using eq. (25) in eq. $(32$,$) , this last becomes$

$$
T_{0}^{L} T_{1}^{L}>\left(R_{1}^{L}\right)^{2}
$$

which is another necessary condition for the positive definiteness of an orthotropic material, see [9]. Finally, condition $(32,3)$ is equivalent to

$$
2 \rho \tau_{1}+\rho \tau_{1}\left(\tau_{0}-1\right)>2,
$$

which is always satisfied because of conditions $\left(26_{2,5}\right)$ and $\left(32_{4}\right)$. This shows that the elastic domain, defined by eq. $(29$ ), always contains the geometric domain, bounded by eq. $(294)$.

An accessory result is that whenever $K^{L}=1$, then $\rho_{1}^{0}>1$; in fact, if $K=K^{L}=1$, $\rho_{1}^{0}>\rho_{1}^{1}>1$, and as $\rho_{1}^{0}$ does not depend upon $K$, it is always greater than 1 . Using once more eq. (25), it is easily checked that

$$
\rho_{1}^{0}>1 \text { for } K^{L}=1 \longrightarrow T_{0}^{L} T_{1}^{L}>2\left(R_{1}^{L}\right)^{2},
$$

which is obvious, by condition $(162)$ when $K^{L}=1$.

The analysis made above concerns the case of ordinary orthotropy, corresponding to eq. (7). The type of the ordinary orthotropy of the material and of the laminate, determined by the value of the coefficients $K^{L}$ and $K$ respectively, appears directly in the equations specifying the bounds, eqs. $\left.22_{4,5}\right)$. This is just an useful peculiarity of the polar method.

In the same way, the polar method allows for directly specifying the existence of a special orthotropy, eqs. (8) and (9). There are four cases of interest for the special orthotropies, that actually correspond to four degenerate cases of eq. (29). Among these four cases, two are those of a laminate composed of a material having a special orthotropy, i.e. having $R_{0}^{L}=0$ or $R_{1}^{L}=0$. In such cases, both tensors $\mathbf{A}^{*}$ and $\mathbf{D}^{*}$ have both the same property, i.e. $R_{0}=0$ or $R_{1}=0$ respectively, as it is apparent from eq. 10 . The other two degenerate cases are those of a laminate composed of ordinary orthotropic layers, but for which tensor $\mathbf{A}^{*}$ or $\mathbf{D}^{*}$ are specially orthotropic, i.e. with $R_{0}=0$ or $R_{1}=0$. In all these cases, the results found above for the ordinary orthotropic case are still valid. In particular, it is easy to check that whenever $R_{0}=0$, the bounds reduce to

$$
0 \leq \rho_{1} \leq \frac{1}{\sqrt{2}} \longrightarrow R_{1} \leq \frac{R_{1}^{L}}{\sqrt{2}}
$$

while for $R_{1}=0$ it is

$$
0 \leq \rho_{0} \leq 1 \rightarrow R_{0} \leq R_{0}^{L}
$$

The above results show also that the geometric conditions bounds more the term $R_{1}$ than the term $R_{0}$. 


\section{Degree of filling of the elastic domain}

We introduce in this section a degree of filling, $\omega$, of the elastic domain of the laminate: we define $\omega$ as the ratio

$$
\omega=\frac{\omega_{g}}{\omega_{e}}
$$

between the airs $\omega_{g}$ and $\omega_{e}$, respectively of the geometric and of the elastic domains defined above.

The ratio $\omega$ measures, in some sense, how much the geometric bounds shrink the elastic domain, giving hence an idea of what is lost, in terms of elastic possibilities, when a material is used to construct a laminate. The air of the geometric domain depends only upon the type of ordinary orthotropy of the material and of the laminate, i.e. upon $K^{L}$ and $K$ : using eqs. $(291)$ to $(29)$ it is easily seen that

$$
\begin{gathered}
\omega_{g}=\frac{4-\sqrt{2}}{3} \simeq 0.862 \quad \text { if } \quad K=K^{L} \\
\omega_{g}=\frac{\sqrt{2}}{3} \simeq 0.471 \quad \text { if } \quad K \neq K^{L} .
\end{gathered}
$$

The air of the elastic domain depends, of course, on the material, see eq. $(295)$; we define it as

$$
\omega_{e}=\rho_{1}^{1}-\int_{\rho_{1}^{0}}^{\rho_{1}^{1}}\left(\frac{2 \rho_{1}^{2}}{\rho \tau_{1}}-\tau_{0}\right) d \rho_{1} \quad \text { if } K=0, \quad \omega_{e}=\rho_{1}^{1}+\int_{\rho_{1}^{1}}^{\rho_{1}^{0}}\left(\tau_{0}-\frac{2 \rho_{1}^{2}}{\rho \tau_{1}}\right) d \rho_{1} \quad \text { if } K=1 .
$$

Nevertheless, a glance at the diagrams in Fig. 1 allows us for giving some bounds on the value of $\omega$ for the four possible cases:

$$
\begin{aligned}
& \text { for } K=0 \text { and } K^{L}=0, \quad \omega_{e} \geq \omega_{g} \longrightarrow \omega \leq 1 \\
& \text { for } K=0 \text { and } K^{L}=1, \quad \omega_{e}>1 \quad \longrightarrow \omega \leq \frac{\sqrt{2}}{3} \simeq 0.471, \\
& \text { for } K=1 \text { and } K^{L}=0, \quad \omega_{e}>\omega_{g} \longrightarrow \omega<1, \\
& \text { for } K=1 \text { and } K^{L}=1, \quad \omega_{e}>1 \quad \longrightarrow \quad \omega<\frac{4-\sqrt{2}}{3} \simeq 0.862 .
\end{aligned}
$$

So, the ratio $\omega$ decreases when $K^{L}=1$.

Let us consider two practical cases; the first one is that of laminates composed of unidirectional carbon-epoxy layers of type T-300/5208, whose elastic properties, [7], are condensed in the reduced stiffness matrix $\mathbf{Q}$ :

$$
\mathbf{Q}=\left[\begin{array}{ccc}
181.8 & 2.89 & 0 \\
2.89 & 10.35 & 0 \\
0 & 0 & 7.17
\end{array}\right] \quad \text { GPa. }
$$

The corresponding polar constants, eq. (6), are

$$
\begin{aligned}
T_{0} & =26.88 \mathrm{GPa}, T_{1}=24.74 \mathrm{GPa}, \\
R_{0} & =19.71 \mathrm{GPa}, R_{1}=21.43 \mathrm{GPa}, \\
\Phi_{0} & =0^{\circ}, \Phi_{1}=0^{\circ}, \longrightarrow K^{L}=0 .
\end{aligned}
$$

Then, injecting these values in eq. 25) we get

$$
\rho=0.92, \tau_{0}=1.36, \tau_{1}=1.15,
$$

which used in eq. $(29$, give the parabolas

$$
(-1)^{K} \rho_{0}=1.89 \rho_{1}^{2}-1.36,
$$


to which correspond

$$
\begin{aligned}
& \rho_{1}^{0}=0.848, \quad \rho_{1}^{1}=1.117, \quad \omega_{e}=0.989, \quad \omega_{g}=0.862, \quad \omega=0.871 \text { for } K=0, \\
& \rho_{1}^{0}=0.848, \quad \rho_{1}^{1}=0.436, \quad \omega_{e}=0.664, \quad \omega_{g}=0.471, \quad \omega=0.709 \text { for } K=1 .
\end{aligned}
$$

The second case is that of laminates whose layers are braided carbon-epoxy plies of the type BR-45a, [1], with reduced stiffness matrix

$$
\mathbf{Q}=\left[\begin{array}{ccc}
55.56 & 20.22 & 0 \\
20.22 & 26.96 & 0 \\
0 & 0 & 25
\end{array}\right] \text { GPa. }
$$

In this case we get, for the polar constants

$$
\begin{gathered}
T_{0}=17.76 \mathrm{GPa}, T_{1}=15.37 \mathrm{GPa}, \\
R_{0}=7.24 \mathrm{GPa}, R_{1}=3.57 \mathrm{GPa}, \\
\Phi_{0}=45^{\circ}, \Phi_{1}=0^{\circ}, \longrightarrow K^{L}=1,
\end{gathered}
$$

for the adimensional parameters

$$
\rho=2.03, \tau_{0}=2.45, \tau_{1}=4.30
$$

for the parabolas

$$
(-1)^{K} \rho_{0}=0.23 \rho_{1}^{2}-2.45
$$

and finally

$$
\begin{aligned}
& \rho_{1}^{0}=3.270, \quad \rho_{1}^{1}=3.880, \quad \omega_{e}=3.577, \quad \omega_{g}=0.471, \quad \omega=0.132 \text { for } K=0, \\
& \rho_{1}^{0}=3.270, \quad \rho_{1}^{1}=2.510, \quad \omega_{e}=2.904, \quad \omega_{g}=0.862, \quad \omega=0.297 \text { for } K=1 .
\end{aligned}
$$

The curves delimiting the elastic and geometric domains and concerning the four cases above are shown in Fig. 2.

\section{The non-orthotropic case}

The results above concern the case of a tensor $\mathbf{A}^{*}$ or $\mathbf{D}^{*}$ orthtropic. We consider in this section the more general case of a tensor that is not orthotropic, though still composed of orthotropic identical layers. This assumption is motivated by the fact that in real applications, the layers composing a laminate are always at least orthotropic. We fix again the frame for the layer and for the laminate by eq. (13), which still implies that $\xi_{3}=0$, condition $(14)$, but not that $\xi_{2}=0$, condition $\left(14_{1}\right)$, because the tensor is no longer orthotropic. So, now there are three geometric parameters, $\xi_{0}, \xi_{1}$ and $\xi_{2}$, for the laminate to be controlled: this will give three dimensional geometric and elastic domains, as specified below.

In this way, eq. (15) becomes

$$
\left\{\begin{array}{l}
R_{0} e^{4 i \Phi_{0}}=(-1)^{K^{L}} R_{0}^{L}\left(\xi_{0}+i \xi_{2}\right), \\
R_{1}=R_{1}^{L} \xi_{1}
\end{array}\right.
$$

and bounds (21) are still valid, as well as bounds (17). Unlike these last, elastic bounds (16) are replaced by

$$
\left\{\begin{array}{l}
T_{0}-R_{0}>0 \\
T_{1}\left(T_{0}^{2}-R_{0}^{2}\right)-2 R_{1}^{2}\left(T_{0}-R_{0} \cos 4 \Phi_{0}\right)>0
\end{array}\right.
$$



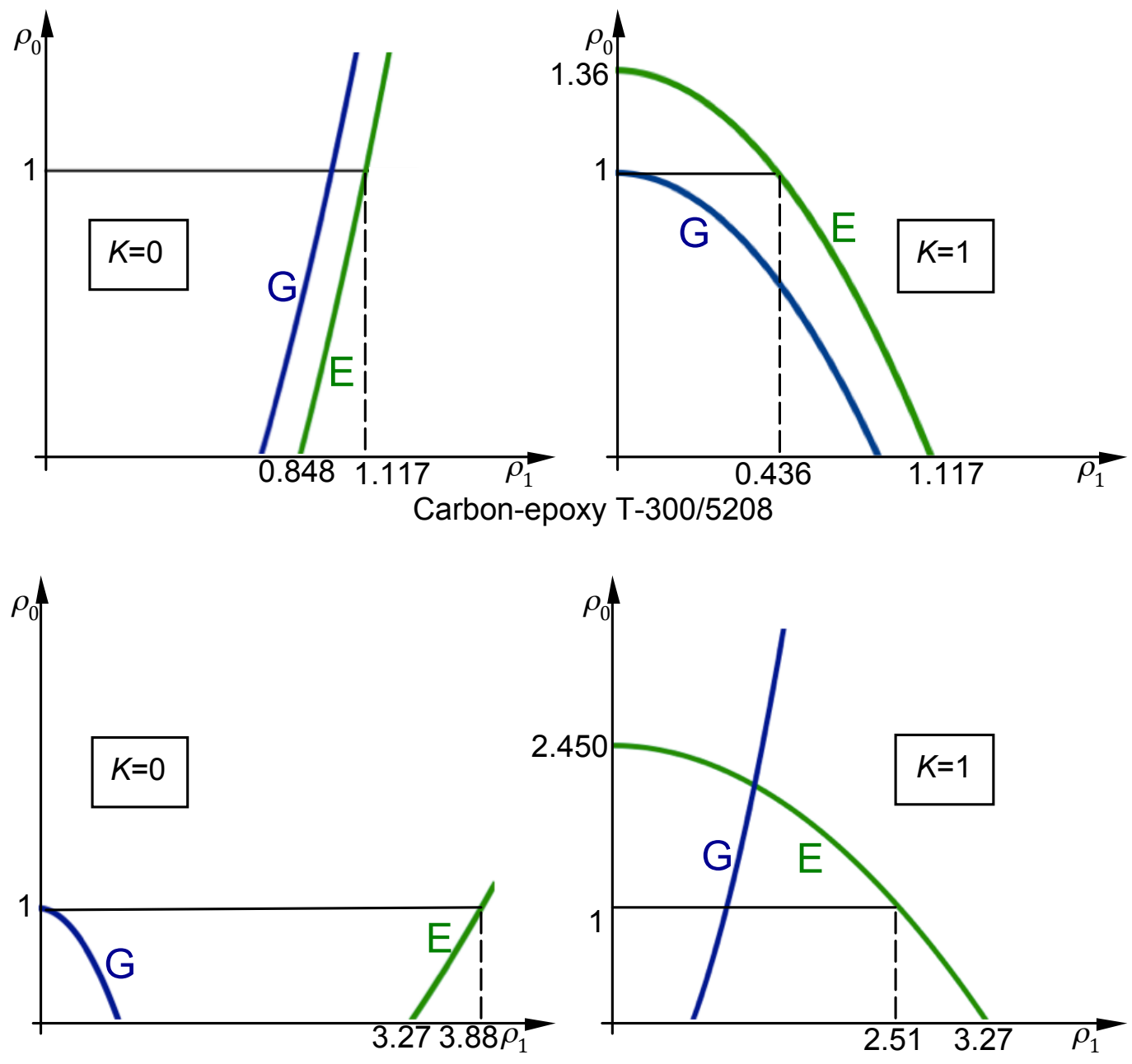

Braided carbon-epoxy BR 45-a

Fig. 2 The geometric and elastic domains relative to the numerical examples considered: the orthotropic cases.

valid for anisotropic tensors, 9], while the geometric bounds $19 p$ become now

$$
\left\{\begin{array}{l}
2 \xi_{1}^{2}\left(1-\xi_{0}\right)+\xi_{0}^{2}+\xi_{2}^{2} \leq 1 \\
\xi_{1}^{2} \leq 1 \\
-1 \leq \xi_{0} \leq 1
\end{array}\right.
$$

because $\xi_{2} \neq 0$.

After introducing once more the dimensionless quantities (25), we get, by eq. (52),

$$
\left\{\begin{array}{l}
\xi_{0}=(-1)^{K^{L}} \rho_{0} \cos 4 \Phi_{0}, \\
\xi_{2}=(-1)^{K^{L}} \rho_{0} \sin 4 \Phi_{0}, \\
\xi_{1}=\rho_{1} .
\end{array}\right.
$$

Using eqs. (25) and (55) into eqs. (17), (19), (53) and (54), and eliminating the redundant 
bounds, likewise in Sec. 4, after some standard passages we get the dimensionless bounds

$$
\left\{\begin{array}{l}
0 \leq \rho_{0}, \\
0 \leq \rho_{1}, \\
\rho_{0} \leq 1, \\
2 \rho_{1}^{2} \leq \frac{1-\rho_{0}^{2}}{1-(-1)^{K L} \rho_{0} c_{0}}, \\
2 \rho_{1}^{2}<\rho \tau_{0} \tau_{1} \frac{1-\left(\frac{\rho_{0}}{\tau_{0}}\right)^{2}}{1-\frac{\rho_{0}}{\tau_{0}} c_{0}} .
\end{array}\right.
$$

that replace eq. $(29)$ for the non orthotropic case. In eq. (56) we have replaced the variable $\Phi_{0}$ by

$$
c_{0}=\cos 4 \Phi_{0}
$$

Now, there are three dimensionless parameters to be taken into account, because with respect to the previous orthotropic case, $c_{0}$ is added to $\rho_{0}$ and $\rho_{1}$. All of them are still invariants: having posed $\Phi_{1}=0, \Phi_{0}$ actually corresponds to the invariant $\Phi_{0}-\Phi_{1}$.

To show that the geometric domain is entirely contained in the elastic one, it is sufficient to show that

$$
\Delta=\rho \tau_{0} \tau_{1} \frac{1-\left(\frac{\rho_{0}}{\tau_{0}}\right)^{2}}{1-\frac{\rho_{0}}{\tau_{0}} c_{0}}-\frac{1-\rho_{0}^{2}}{1-(-1)^{K^{L}} \rho_{0} c_{0}}>0 \quad \forall \rho_{0} \in[0,1], c_{0} \in[-1,1], \rho \tau_{0} \tau_{1}>1, \tau_{0}>1 .
$$

Considering the values taken by $\Delta$ on the boundary of the domain defined in eq. (58) and in the only point, $\rho_{0}=0, c_{0}=0$, where grad $\Delta=0$, it can be easily seen that the absolute minima of $\Delta$ can be only:

$$
\begin{aligned}
& \Delta_{1}^{\text {min }}=\rho \tau_{0} \tau_{1}-1, \quad \text { for } \rho_{0}=0,-1 \leq c_{0} \leq 1 \\
& \Delta_{2}^{\text {min }}=\rho \tau_{1}\left(\tau_{0}-1\right), \quad \text { for } \rho_{0}=1, c_{0}=-1
\end{aligned}
$$

Both these values are positive, by virtue of eqs. (26) and $(32$ ) and this proves the condition (58).

Also in this case we can introduce a degree of filling of the elastic domain of the laminate, $\omega$, defined just like in eq. (38), but now it is

$$
\omega_{g}=\int_{0}^{1} \int_{-1}^{1} \sqrt{\frac{1-\rho_{0}^{2}}{2\left(1-(-1)^{K^{L}} \rho_{0} c_{0}\right)}} d \rho_{0} d c_{0}, \omega_{e}=\int_{0}^{1} \int_{-1}^{1} \sqrt{\rho \tau_{0} \tau_{1} \frac{1-\left(\frac{\rho_{0}}{\tau_{0}}\right)^{2}}{2\left(1-\frac{\rho_{0}}{\tau_{0}} c_{0}\right)}} d \rho_{0} d c_{0}
$$

In Fig. 3 the two numerical examples, considered in the previous section, of carbon-epoxy T-300/5208 and braided carbon-epoxy BR45-a in the general anisotropic case are shown; once more, $\mathrm{G}$ and $\mathrm{E}$ denote the boundaries of the geometric and of the elastic domains respectively. Numerically, we obtain $\omega=0.709$ for the T-300/5208 and $\omega=0.296$ for the BR45-a.

\section{Final remarks}

We have proved in this paper that the elastic domain of a tensor describing the behaviour of a laminate always entirely contains the geometric domain. Mechanically, this means that laminates constitute a sort of smaller elastic class, in the sense that they never can cover 


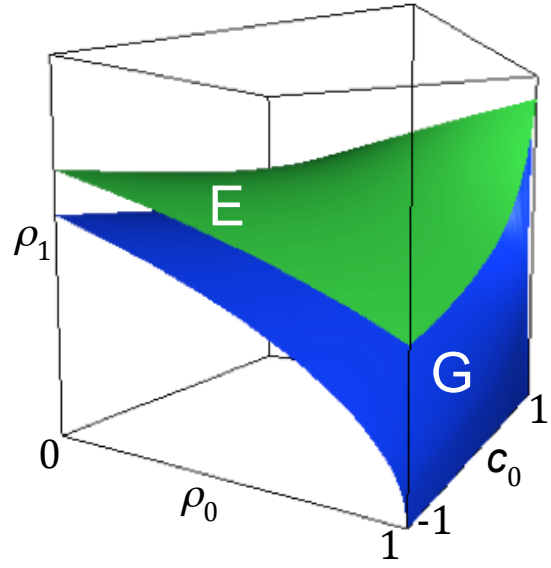

Carbon-epoxy T-300/5208

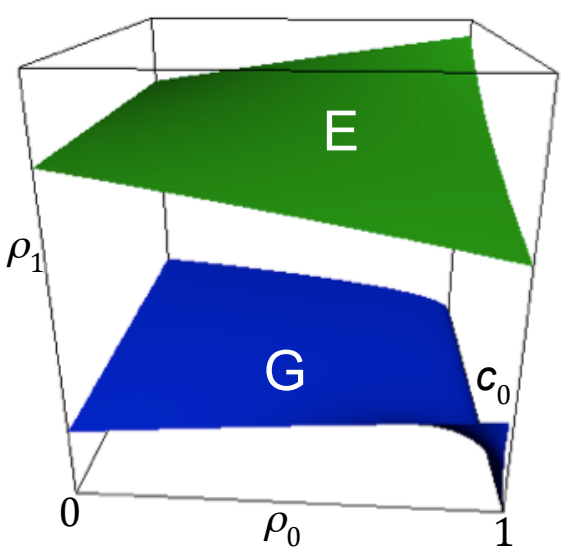

Braided carbon-epoxy BR45-a

Fig. 3 The geometric and elastic domains relative to the numerical examples considered: general anisotropic case.

the whole range of the elastic parameters that can be covered by a single elastic layer. In addition, a measure quantifying the shrinkage of the elastic domain has been given.

All the analysis is based upon the use of the polar formalism, that has several advantages. Namely, it allows for an analysis making use only of invariant quantities, which is particularly important in the definition of the different bounds. Then, with the polar formalism the different elastic invariants of the laminate depend only upon the corresponding quantity of the material (e. g. $R_{0}$ is a function of $R_{0}^{L}$ alone and so on) and the isotropic part is separated form the anisotropic one. Thanks to this last property and to eq. (10), it is easily seen that the shrinkage of the elastic domain for the laminate concerns only its anisotropic part, while the isotropic one is exactly the same of the material and hence has the same bounds. The use of the polar formalism has also allowed for writing in a unique space of invariant dimensionless parameters the elastic and geometrical bounds and offered a new physical interpretation of the classical lamination parameters.

This study is interesting per se, because it mathematically shows and quantifies something that has always been, more or less, tacitly understated in several studies on laminates. It is particularly important in some procedures of optimization of laminated structures, like those presented, for instance, in [3]. In such procedures, the field of the anisotropic constants is optimized in a first step, with respect to an objective and with some imposed constraints, as the one of a structure composed by a fictitious unique anisotropic layer. Then, in a second step, a laminate realizing the optimal anisotropic field found in step one is looked for. So, the present study shows that among the constraints to be taken into account in the first step of the procedure, only the geometric bounds, and not the elastic ones, are to be taken into account among the constraints to be imposed to the optimum. In fact, if elastic constants were imposed in place of the geometric ones, one could perhaps find an optimal anisotropic field with elastic parameters that could not be really obtained by a laminate during the following second step. In order to avoid such a possibility, geometric bounds are to be imposed directly to the optimum problem of the first step.

The results found in the paper are valid for both tensors $\mathbf{A}^{*}$ and $\mathbf{D}^{*}$ taken separately. The case of stating bounds for the whole behaviour of the laminate, i.e. for $\mathbf{A}^{*}$ and $\mathbf{D}^{*}$ at 
the same time, has not been treated in this paper. Actually, up till now no global geometric bounds are known for the lamination and polar parameters of $\mathbf{A}^{*}$ and $\mathbf{D}^{*}$ taken together. It is obvious that these parameters are not independent, because they depend upon a unique stacking sequence. However, it seems very hard to state such bounds because, unlike those of $\mathbf{A}^{*}$, the parameters of $\mathbf{D}^{*}$ depend also on the stacking position, not only on the orientation angle of the layers.

\section{References}

[1] Falzon, P.J., Herszberg, I.: Mechanical performance of 2-d braided carbon/epoxy composites. Composites Science and Technology 58, 253-265 (1998)

[2] Hammer, V.B., Bendsoe, M.P., Lipton, R., Pedersen, P.: Parametrization in laminate design for optimal compliance. International Journal of Solids and Structures 34, 415-434 (1997)

[3] Jibawy, A., Julien, C., Desmorat, B., Vincenti, A., Léné, F.: Hierarchical structural optimization of laminated plates using polar representation. International Journal of Solids and Structures 48, 2576-2584 (2011)

[4] Jones, R.M.: Mechanics of composite materials. McGraw-Hill (1975)

[5] Miki, M.: Material design of composite laminates with required in-plane elastic properties. pp. 1725-1731. ICCM 4 (Fourth International Conference on Composite Materials), Tokio (1982)

[6] Miki, M.: Design of laminated fibrous composite plates with required flexural stiffness. In: J.R. Vinson, M. Taya (eds.) Recent advances in composites in the USA and Japan ASTM STP 864, pp. 387-400. American Society for Testing and Materials, Philadelphia (1985)

[7] Tsai, S.W., Hahn, T.: Introduction to composite materials. Technomic (1980)

[8] Vannucci, P.: A special planar orthotropic material. Journal of Elasticity 67, 81-96 (2002)

[9] Vannucci, P.: Plane anisotropy by the polar method. Meccanica 40, 437-454 (2005)

[10] Vannucci, P.: Influence of invariant material parameters on the flexural optimal design of thin anisotropic laminates. International Journal of Mechanical Sciences 51, 192-203 (2009)

[11] Vannucci, P.: On special orthotropy of paper. Journal of Elasticity 99, 75-83 (2010)

[12] Vannucci, P., Verchery, G.: Stiffness design of laminates using the polar method. International Journal of Solids and Structures 38, 9281-9294 (2001)

[13] Verchery, G.: Les invariants des tenseurs d'ordre 4 du type de l'élasticité. Colloque Euromech 115, Editions du CNRS, Villard-de-Lans (1979) 